\title{
Energy consideration in processes of transition to equilibrium state
}

\author{
Sami M. Al-Jaber ${ }^{1}$, Abdel-Rahman M. Abu-Labdeh ${ }^{2}$ \\ ${ }^{1}$ Department of Physics, An-Najah National University, Nablus, Palestine; Corresponding Author: jaber@najah.edu \\ ${ }^{2}$ Department of Physics, Arab American University, Jenin, Palestine; alabdeh@aauj.edu
}

Received 25 December 2010; revised 26 January 2011; accepted 29 January 2011.

\begin{abstract}
A calculation of the energy loss due to a transition from non-equilibrium to equilibrium state is given. Three different physical systems are considered in this study: connecting an uncharged capacitor to a charged capacitor, emission of a photon from an excited atom, and releasing an object from a compressed spring. In this study, it is shown that a specific fraction of the total energy stored in a non-equilibrium system should be consumed to reach the equilibrium state.
\end{abstract}

Keywords: Equilibrium State; Non-Equilibrium State; Energy Loss; Transition; Two-Capacitor Problem; Emission of a Photon; Compressed Spring

\section{INTRODUCTION}

It is well known that energy is a very important fundamental concept in physics [1]. On one hand, energy leads to describe and understand many processes around us. On the other hand, it has many important applications in different aspects of life. For simplicity, energy can be subdivided and classified in different forms: kinetic energy, potential energy, electromagnetic energy, nuclear energy, etc. The conservation of energy is one of the most important laws of physics [2,3]. According to the law of conservation of energy, energy can only be transformed from one form to another, such as when chemical energy is transformed into electric energy. Energy transformation, usually termed as energy conversion, is very important in the application of energy as well as in natural sciences.

The only way the energy of a given system can change is by carrying energy, in several ways, into or from that system. Energy is therefore a measure of the ability of a given system to produce changes in its own state as well as changes in the states of its surroundings [4]. It follows that a transition from the non-equilibrium to equilibrium states for a given system can occur by changing its initial energy. This is consistent with previous works, where it was pointed out that the stored energy of one kind in a given system can not be transferred without loss unless it undergoes a change in energy form in the transfer process [5]. So in a process of transition to the equilibrium state some amount of the initial energy stored in a given non-equilibrium system must be lost (i.e., transferred to its surroundings) in one form or another. One of the well-known examples of the energy loss in a process of a transition to the equilibrium state is the connection of an uncharged capacitor to a charged capacitor (or simply, the two-capacitor problem) [6-9].

A deeper look into the energy loss in processes of transition to equilibrium states is often missing. In addition, authors (especially in introductory physics textbooks $[10,11])$ do not shed enough light on problems involving such processes. Moreover, authors usually consider ideal cases in problems that are dealing with transformations of energy. For example, in most problems involving the elastic potential energy stored in a stretched or compressed spring in introductory physics textbooks, it is assumed that the end of the spring is fixed, and thus the recoiling kinetic energy in such problems is negligible.

The aim of this study is therefore to show that a specific fraction of the initial energy stored in a given non-equilibrium system must be consumed, in one form or another, to achieve an equilibrium state. The layout of the paper is as follows. In the following section we derive and present the energy loss in the process of a transition from non-equilibrium to equilibrium states for three different physical systems: charging a capacitor by connecting it to charged capacitor, emission of a photon from an excited atom, and releasing an object from a compressed spring. We close the article by summarizing the results and discussing their significance.

\section{CALCULATIONS AND DISSIONS}

In this section, the derivation of the energy loss due to 
a transition from non-equilibrium to equilibrium state for each system of interest is given. We start our calculations on the two-capacitor problem, then on emission of a photon from an excited atom, and finally on releasing an object from a compressed spring. Throughout, discussions of the final results are presented.

\subsection{The Two-Capacitor Problem}

Consider a capacitor of capacitance $C_{1}$ charged to voltage $V o$, which is connected to an uncharged capacitor of capacitance $C_{2}$ by an open switch and long cylindrical metallic wire of length $L$ and radius $a$. The initial electrostatic energy $U_{0}$ stored in the system is therefore given by

$$
U_{0}=\frac{1}{2} C_{1} V_{0}^{2} .
$$

After closing the switch, the system reaches the equilibrium state by the conservation of charge and the requirement for a common final potential difference across the two capacitors. At equilibrium, the common potential $V$ and electrostatic energy $U$ stored in the system are

$$
V=\frac{C_{1}}{C_{1}+C_{2}} V_{0},
$$

and

$$
U=\frac{C_{1}}{C_{1}+C_{2}} U_{0},
$$

so that the difference in electrostatic energy between the initial and final states $\Delta U$ (i.e., the energy loss) is

$$
\Delta U=U-U_{0}=\frac{C_{2}}{C_{1}+C_{2}} U_{0} .
$$

Eq.4 shows that charging a capacitor causes energy loss regardless of the Nature of the dissipative elements in the circuit. The question now is: where does the energy loss go? The easiest answer, which is traditionally cited in standard introductory physics textbooks $[10,11]$, is that it transforms to heat due to electrical resistance in the connecting wires. Suppose that no dissipative elements are apparent in the circuit (i.e., zero-resistance circuit), again where does the loss in the energy go? To answer the question, let the charge on the first capacitor, at any time $t$ between the initial and the final states, is $q_{1}(t)$ and on the second capacitor is $q_{2}(t)$. Thus, the current in the circuit at any time $t$ is

$$
I(t)=\frac{d q_{2}}{d t}=-\frac{d q_{1}}{d t} .
$$

If the total charge on the system is $Q_{0}$, then

$$
q_{2}(t)=Q_{0}-q_{1}(t),
$$

and hence the electric field parallel to the connecting wire is given by

$$
\vec{E}=\frac{1}{L}\left[\frac{Q_{0}-q_{2}(t)}{C_{1}}-\frac{q_{2}(t)}{C_{2}}\right] \hat{Z} .
$$

The magnetic field $\vec{B}$ at the surface of the wire is $[22,24]$

$$
\vec{B}=\frac{\mu_{0} I(t)}{2 \pi a} \hat{\phi}
$$

Substituting Eq.5 into Eq.8 gives

$$
\vec{B}=\frac{\mu_{0}}{2 \pi a} \frac{d q_{2}(t)}{d t} \hat{\phi} .
$$

According to the pointing theorem $[23,24]$, the electromagnetic energy $U_{e m}$ flow into the wire through its closed surface is

$$
U_{e m}=-\int\left[\oint\left(\vec{E} \times \frac{\vec{B}}{\mu_{0}}\right) \cdot d \vec{A}\right] d t .
$$

From Eqs.7 and 9, Eq.10 then gives

$$
U_{e m}=\frac{Q_{2} Q_{0}}{C_{1}}-\frac{Q_{2}^{2}}{2 C_{1}}-\frac{Q_{2}^{2}}{2 C_{2}}=Q_{2} V_{0}-\frac{Q_{2}^{2}}{2 C_{1}}-\frac{Q_{2}^{2}}{2 C_{2}},
$$

where $Q_{2}$ is the equilibrium charge on the second capacitor, which is given by

$$
Q_{2}=\frac{C_{1} C_{2}}{C_{1}+C_{2}} V_{0} .
$$

Using Eq.12, $U_{e m}$ then becomes

$$
U_{e m}=\left(\frac{C_{2}}{C_{1}+C_{2}}\right) \frac{1}{2} C_{1} V_{0}^{2}=\frac{C_{2}}{C_{1}+C_{2}} U_{0}
$$

which is the energy loss given by Eq.4. Eq.13 gives the electromagnetic energy that flows into the connecting wire and this indicates that a specific fraction of the initial energy stored in the system flows into the connecting wire through its closed surface to achieve the final equilibrium state. It is clear that when the two capacitors have the same capacitance then the energy loss is exactly half the initial stored energy. As indicated by Eq.4, the energy loss is independent of the value of the electrical resistance. Some authors showed that if no dissipative elements are present in the circuit, the energy loss is radiated as electromagnetic energy [12-16]. Recent work related to energy transfer and its connection to nanotechnology and advanced materials had been reported $[17,18]$. 


\subsection{Emission of a Photon from an Excited Atom}

Let us now look to another non-equilibrium system. This system is a stationary excited atom of energy $E_{i}$ and mass $M$ [19]. To reach the equilibrium state, the energy of the atom decreases to $E_{f}$ by emitting a photon of frequency $v$. So the energy difference $\Delta E$ between the two states of the atom is

$$
\Delta E=E_{i}-E_{f}
$$

Because the atom is assumed to be initially at rest, so that the conservation of linear momentum requires

$$
\vec{P}_{\text {photon }}=-\vec{P}_{\text {atom }}
$$

Eq.15 tells us that the emitted photon and recoiling atom should have the same magnitude of linear momentum $P=h v / c$, where $h$ is the Planck's constant and $c$ is the speed of light. Based on Eq.15, the conservation of energy is given by the expression

$$
E_{i}=E_{f}+h v+\frac{P_{a t o m}^{2}}{2 M}=E_{f}+h v+\frac{(h v)^{2}}{2 M c^{2}},
$$

where $h v$ is the energy of the emitted photon and $P_{\text {atom }}^{2} / 2 M$ is the kinetic energy of the recoiling atom. By solving Eq.16, the energy of the emitted photon is therefore

$$
h v=M c^{2}\left[-1+\left(1+\frac{2 \Delta E}{M c^{2}}\right)^{1 / 2}\right] .
$$

$\Delta E$ is usually much less than the rest energy of the atom $M c^{2}$, so that Eq.17 gives

$$
h v=\Delta E-\frac{(\Delta E)^{2}}{2 M c^{2}},
$$

or

$$
\frac{(\Delta E)^{2}}{2 M c^{2}}=\Delta E-h v .
$$

Eq.19 shows that the majority of the transition energy generated by the excited atom is "stored" in the emitted photon and the reminder of it is given to the recoiling atom as a kinetic energy. Therefore, an exited atom can make a transition from a higher energy state (non-equilibrium state) to a lower energy state (equilibrium state) by emitting a photon. To conserve both energy and momentum, the atom must recoil when it emits such a photon $[20,21]$.

Because the kinetic energy of the recoiling atom is very small compared to the rest energy, it is usually ignored in physics textbooks. Although this kinetic energy is tiny, it has subtle effect in various applications and natural sciences. For example, recoiling atom has an important effect on the frequency of the spontaneously emitted photons in resonance fluorescence processes [24].

\subsection{Releasing an Object from a Compressed Spring}

Analogous to the previous system, the following example shows how the elastic potential energy stored in a compressed spring is transformed when a transition from non-equilibrium to equilibrium states occurred. In this example, consider an object of mass $m$ which is attached to the end of a compressed spring of negligible mass. The other end of the spring is assumed to be connected with another object of mass $M$. Let us also assume that the system is initially at rest and is placed on a frictionless surface.

When the object $m$ is released from rest the spring snaps back to its original length and then the conservation of linear momentum requires

$$
\vec{P}_{m}=-\vec{P}_{M}
$$

where $P_{m}$ and $P_{M}$ are, respectively, the magnitude of the linear momentum for the mass $m$ and the recoiling mass $M$. So that the conservation of energy is given by the expression

$$
\begin{aligned}
U_{S} & =\frac{P_{m}^{2}}{2 m}+\frac{P_{M}^{2}}{2 M} \\
& =\frac{P_{m}^{2}}{2 m}+\frac{P_{m}^{2}}{2 M} \\
& =\frac{P_{m}^{2}}{2 m}\left(1+\frac{m}{M}\right),
\end{aligned}
$$

or

$$
\frac{P_{m}^{2}}{2 m}=\frac{M}{m+M} U_{S},
$$

where $U_{S}$ is the elastic potential energy stored into the compressed spring. In a similar fashion to the process of emitting a photon from an exited atom, Eq.22 shows that a fraction of the total energy stored into the spring is given to the released object $m$ and the reminder of it is given to the recoiling mass $M$ as a kinetic energy. One may also notice that when the two masses are equal then only half of the initial stored energy will be given to the released mass $m$ while the other half has been given to the recoil mass $M$.

\section{CONCLUSIONS}

In this paper we derive the energy loss in the process 
of a transformation from non-equilibrium to equilibrium states for three different physical systems. In the two capacitor problem, the calculations show that a specific fraction of the initial electrostatic energy stored in a charged capacitor is lost as an electromagnetic energy, which passes into the connecting wire through its closed surface. In the process of emitting a photon from an excited atom, the calculations show that the majority of the energy difference between the equilibrium and nonequilibrium states is emitted from the excited atom as a photon energy and the reminder of the total energy is given to the recoiling atom as a kinetic energy. An analogy to the situation of an exited atom, the results indicate that part of the total elastic potential energy stored in a compressed spring is given to the released object and the reminder of it is left to the recoiling system as a kinetic energy.

Analogous situations persist in two widely wellknown examples in introductory physics courses [10,11]. The first is that of a uniform loop which is rolling without slipping on an inclined plane. The physics conclusion here is that, due to the non-slip constraint, the loop rolls at one half of the speed it would exhibit if it could slip freely (i.e., if non of the potential energy went into the rolling kinetic energy). In this example, as it is well known, the translational kinetic energy at the end of the inclined plane is half of the initial potential energy and the other half went as rolling kinetic energy due to the non-slip constraint.

The second example is the total work required to charge a spherical conducting shell of radius $a$ to a total charge $Q$. A typical problem in most standard textbooks gives an answer of $Q^{2} /\left(8 \pi \varepsilon_{0} a\right)$ (for example, see problem 59 of chapter 25 in reference [10]). This amount of energy should be the electrostatic energy stored in the electric field of the charged spherical shell not the total work required to charge the spherical shell. A closer look to this problem shows that this is just charging an isolated spherical capacitor, and according to our result presented above the total work that must be done to charge the spherical shell is just twice the stored energy (namely, $Q^{2} / 4 \pi \varepsilon_{0} a$ ). Therefore, problems involving such examples in standard introductory textbooks, in particular, and in electromagnetism textbooks, in general, are sometimes miss-leading the students because, in reality, the process of charging an object should be done through a conducting medium which connects the object with the power source as seen in the process of charging a capacitor presented above. In order to avoid any confusions in physics concepts, we think that it would be better (in such problems) to ask students to calculate the stored electrostatic energy in the electric field of the charged object rather than the work that must be done to charge that object.

We must emphasize that in all the above discussed systems, a constraint has been imposed on each of them in the process of transition from non-equilibrium to equilibrium states. In a process of charging a capacitor, for example, authors of physics textbooks usually assumed that the conducting wires has an electrical resistance and all energy loss in this process is dissipated as heat in that resistance. Analogously, in the case of an excited atom and elastic compressed spring, it is usually assumed that the effect of recoil is negligible.

In addition, our results may form a good basis to explain the reason behind the usual assumption for the non-conservation of kinetic energy in the completely inelastic collisions in which the two colliding particles before collision (non-equilibrium) become one particle after collision (equilibrium) by the common velocity requirement as a constraint. A mechanism must be needed to mediate the constraint to the system in order to make a transition from non-equilibrium to equilibrium state. This is in contrast to elastic processes in which no constraint is imposed on the system, and thus there is no need to such a mechanism and therefore there is no loss in the initial kinetic energy of the system. As a result the present authors believe that this is the justification why physicists usually state conservation of kinetic energy as a priori when dealing with elastic collisions. However, consumed energy (in one form or another) is actually the price that should be paid in order to achieve the final equilibrium state.

\section{REFERENCES}

[1] Brown, T.M. (1965) Resource letter EEC-1 on the evolution of energy concepts from Galileo to Helmholtz. American Journal of Physics, 33, 759-765.

doi:10.1119/1.1970980

[2] Plasson, R. and Brandenberg, A. (2010) Homochirality and the need for energy. Origin of Life and Evolution Biospheres, 40, 93-110. doi:10.1007/s11084-009-9181-6

[3] Aubrecht, G.J. (1995) Energy. Prentice Hall, New Jersey.

[4] Haddad, W.M., Chellaboina, V. and Nersesov, S.G. (2005) Thermodynamics: A dynamical systems approach. Prineton University Press, New Jersey.

[5] O'Connor, W.J. (1997) The famous 'lost' energy when two capacitors are joined: A new law? Physics Education, 32, 88-90. doi:10.1088/0031-9120/32/2/014

[6] Powell, R.A. (1979) Two-capacitor problem: A more realistic view. American Journal of Physics, 47, 460-463. doi:10.1119/1.11817

[7] Mould, S. (1998) The energy lost between two capacitors: An analogy. Physics Education, 33, 323-326. doi:10.1088/0031-9120/33/5/018

[8] Al-Jaber, S.M. and Salih, S.K. (2000) Energy consideration in the two-capacitor problem. Europe Journal of 
Physics, 21, 341.

doi:10.1088/0143-0807/21/4/307

[9] Abu-Labdeh, A.M. and Al-Jaber, S.M. (2008) Energy consideration from non-equilibrium to equilibrium state in the process of charging a capacitor. Journal of Electrostatics, 66, 190-192. doi:10.1016/i.elstat.2007.12.002

[10] Serway, R. (2006) Physics for scientists and engineers with modern physics. Saunders, London.

[11] Halliday, D., Resnick, R. and Walker, J. (2005) Fundamental of physics. John Wiley and Sons, New York.

[12] Mayer, R.P., Jeffries, J.R. and Paulik, G.F. (1993) The two-capacitor problem reconsidered. IEEE Transactions on Education, 36, 307-309. doi:10.1109/13.231509

[13] Timothy, B.B., Hite, D. and Singh, N. (2002) The twocapacitor problem with radiation. American Journal of Physics, 70, 415-420. doi:10.1119/1.1435344

[14] Choy, T.C. (2004) Capacitors can radiate: Further results for the two-capacitor problem. American Journal of Physics, 72, 662-670. doi:10.1119/1.1643371

[15] Mita, K. and Boufaida, M. (1999) Ideal capacitor circuits and energy conservation. American Journal of Physics, 67, 737-738. doi:10.1119/1.19363
[16] Wallace, G.G., Chen, J., Mozer, A.J., Forsyth, M. Macfarlane, D.R. and Wang, C. (2009) Nanoelectrodes: Energy conversion and storage. Materials Today, 12, 2027. doi:10.1016/S1369-7021(09)70177-4

[17] Pardos, A., Bonilla, L.L. and Carpio, A. (2010) Phase transitions in a mechanical system coupled to glauber spins. Journal of Statatical Mech: Theory and Experiment, June 2010, P06016.

[18] Liu, C., Li, F., Ma, L. and Cheng, H. (2010) Advanced materials for energy storage. Advanced Materials, 22, E28-E62. doi:10.1002/adma.200903328

[19] Brehm, J.J. and Mullin, W.J. (1989) Introduction to the structure of matter. John Wiley and Sons, New York.

[20] Wasson, T. (1987) Nobel prize winners: An biographical dictionary. Wilson, H.W., University of Michigan, Michigan.

[21] Niemantsverdriet, J.W. (2000) Spectroscopy in catalysis: an introduction. Wiley-VCH Verlag $\mathrm{GmbH}$ and Co. KgaA, Weinheim. doi:10.1002/9783527614127

[22] Lam, J.F. and Berman, P.R. (1976) Effect of recoil in resonance fluorescence. Physical Review A, 14(5), 16831688 . doi:10.1103/PhysRevA.14.1683

[23] Griffiths, D.J. (1999) Introduction to electrodynamics. Prentice-Hall, New Jersey.

[24] Nayfeh, M.H. and Brussel, M.K. (1985) Electricity and magnetism. John Wiley and Sons, New York. 